\title{
Uniform Convergence of Extremal Polynomials When Domains Have Corners and Special Cusps on the Boundary
}

\author{
Cem Koşar ${ }^{1}$, Mehmet Kucukaslan ${ }^{2}$, Fahreddin G. Abdullayev ${ }^{2}$ \\ ${ }^{1}$ Department of Mathematics, Faculty of Science and Literature, Bitlis Eren University, Bitlis, Turkey \\ ${ }^{2}$ Department of Mathematics, Faculty of Science and Literature, Mersin University, Mersin, Turkey \\ E-mail: ckosar77@gmail.com, \{mkucukaslan,fabdul\}@mersin.edu.tr \\ Received May 26, 2011; revised September 13, 2011; accepted September 22, 2011
}

\begin{abstract}
We study the approximation properties of the extremal polynomials in $A_{p}$-norm and C-norm. We prove estimates for the rate of such convergence of the sequence of the extremal polynomials on domains with corners and special cusps.
\end{abstract}

Keywords: Uniform Approximation, Extremal Polynomials, Conformal Mapping, $p$-Bieberbach Polynomials

\section{Introduction and Main Results}

\subsection{Statement of Problem and Some Definitions}

Let $G \subset \mathbb{C}$ be a finite domain bounded by Jordan curve $L:=\partial G$ and $\zeta_{0} \in G$ be an arbitrary fixed point. Let $w=\varphi(z)$ be conformal mapping of $G$ onto the disk $D\left(0, r_{0}\right):=\left\{w:|w|<r_{0}\right\}$ with normalization $\varphi\left(\zeta_{0}\right)=0, \quad \varphi^{\prime}\left(\zeta_{0}\right)=1$, where $r_{0}$ is a conformal radius of $G$ with respect to $\zeta_{0}$ and let $\psi:=\varphi^{-1}$ be an inverse mapping.

Let $p>0$. We denote by $A_{p}^{1}(G)$ the set of functions $f(z)$ analytic in $G$ and normalized by $f\left(\zeta_{0}\right)=0, \quad f^{\prime}\left(\zeta_{0}\right)=1$ such that

$$
\|f\|_{A_{p}^{1}(G)}:=\left(\iint_{G}\left|f^{\prime}(z)\right|^{p} \mathrm{~d} \sigma_{z}\right)^{\frac{1}{p}}<\infty,
$$

where $\mathrm{d} \sigma_{z}$ is a two-dimensional Lebesque measure on G.

We denote by $\wp_{n}$ of all algebraic polynomials

$P_{n}(z), \operatorname{deg} P_{n} \leq n$ satisfying $P_{n}\left(\zeta_{0}\right)=0, \quad P_{n}^{\prime}\left(\zeta_{0}\right)=1$.

Let us consider following extremal problem:

$$
\left\{\left\|\varphi-P_{n}\right\|_{A_{p}^{1}(G)}: P_{n} \in \wp_{n}\right\} \rightarrow \min
$$

Using a method similar to the one given in ([1], p. $137)$, it is seen that there exists an extremal polynomial
$P_{n}^{*}(z)$ furnishing to the problem (1.1), these polynomials $P_{n}^{*}(z)$ are determined uniquely in case of $p>1$ ([1], p. 142). This extremal problem was first considered by Kucukaslan M. and Abdullayev F.G. and they were called the $p$-Bieberbach polynomial of degree $n$ for the pair $\left(G, \zeta_{0}\right)$ in [2], and denoted by $B_{n, p}(z)$.

The main goal of this paper is to investigate the approximation rate of convergence $B_{n, p}(z)$ to the function $\varphi$ in uniform norm for some domains when has some certain singularity, i.e.

$$
\left\|\varphi-B_{n, p}\right\|_{C(\bar{G})}:=\max _{z \in \bar{G}}\left\{\left|\varphi(z)-B_{n, p}(z)\right|\right\} \leq \frac{c}{n^{\gamma}},
$$

where $\gamma:=\gamma(p, G)>0$, the constant $c$ is independent of $n$.

In case of $p=2$, the solution of (1.1) coincides with the well known $n$-th Bieberbach polynomial $B_{n}(z) \equiv B_{n, 2}(z)$ (see, for example, [3,4] ). The approximation properties in the uniform norm of $B_{n}(z)$ on $\bar{G}$ first was observed by Keldysh in 1939 [3] for the domains with sufficiently smooth boundary. A considerable progress in this area has been achieved by Mergelyan [5], Suetin [4], Simonenko [6], Andrievskii [7,8] Gaier [9,10], Abdullayev [11-13], Israfilov [14,15] and the others.

In this paper, we are going to consider the case $p>1$ the problem in (1.2). First, we will investigate the approximation rate of $B_{n, p}(z)$ to the function $\varphi$ in 
$A_{p}^{1}$ - norm and using well known Simonenko and Andrievskii method (see, for example, [6,7]), the approximation rate of $B_{n, p}(z)$ to the function $\varphi$ in uniform norm will be obtained.

Now we need some definitions:

Definition 1.1. ([16], p. 97)The Jordan arc or a curve $L$ is called a $K$ - quasiconformal $(K \geq 1)$ arc or curve if there is a $K$-quasiconformal mapping $f$ of a region $D$ containing $L$ such that $f(L)$ is line segment or circle.

Let $F(L)$ denote the set of all sense-preserving plane homeomorphisms $f$ of regions $D \supset L$ such that $f(L)$ is a line segment or circle and let

$$
K(L)=\inf \{K(f): f \in F(L)\},
$$

where $K(f)$ is the maximal dilatation of such a mapping $f$. Then $L$ is $K$-quasiconformal if and only if $K(L)<\infty$. If $L$ is a $K$-quasiconformal, then $K(L) \leq K$.

$D \equiv \overline{\mathbb{C}}$ gives the global definition of a $K$ - quasiconformal arc or curve consequently. This definition is common in the literature. At the same time, we can consider the domain $D \supset L$ as the neighborhood of the curve $L$. In this case, Definition 1.1 will be called local definition of quasiconformal arc or curve. Through this work we consider the local definition. The local definition has an advantage in determining the coefficients of quasiconformality for some simple arcs or curves.

Let us denote $z=z(s), \quad s \in[0$, mesL $]$ natural representation of $L:=\partial G$.

Definition 1.2. ([4]) We say that $G \in C_{\theta}$ if $\partial G$ has a continuous tangent $\theta(s):=\theta(z(s))$ for every points $z(s)$.

Corollary 1.1. ([17]) If $G \in C_{\theta}$, then $K=1+\varepsilon$ for all $\varepsilon>0$.

Definition 1.3. ([12]) We say that $G \in C_{\theta}(\lambda ; \alpha), 0<\lambda<2, \alpha \geq 0$, if $L:=\partial G$ is expressed as a union of a finite number of $C_{\theta}$-arcs, connecting at the points $Z_{0} Z_{1}, \cdots, Z_{m}$, such that $L$ is locally smooth at $z_{0}$ and in the local coordinate system $(x, y)$ with origin at $z_{j}, \quad 1 \leq j \leq m$, the following conditions are satisfied:

1) For every $z_{j}, \quad 1 \leq j \leq p \leq m$, the domain $G$ has $\lambda_{j} \pi, \quad 0<\lambda_{j}<2$, exterior angle at the corner $z_{j}$, $\min \left\{\lambda_{j}\right\}=: \lambda$.

2) For every $z_{j}, \quad p+1 \leq j \leq m$, in $(x, y)$ coordinate system with origin at $z_{j}$ we have

$$
\begin{gathered}
\left\{z=x+i y: \tilde{c}_{1} x^{1+\alpha} \leq y \leq \tilde{c}_{2} x^{1+\alpha}, 0 \leq x \leq \varepsilon_{1}\right\} \subset \bar{G}, \\
\left\{z=x+i y:|y| \geq \varepsilon_{2} x, 0 \leq x \leq \varepsilon_{1}\right\} \subset C G,
\end{gathered}
$$

for some constants $-\infty<\tilde{c}_{1}<\tilde{c}_{2}<\infty, \quad \varepsilon_{i}>0, \quad i=1,2$.

It is clear from the definition that each domain $G \in C_{\theta}(\lambda ; \alpha)$ may have exterior $\lambda_{j} \pi$ angles, $0<\lambda_{j}<2$, at the points $z_{j}, 1 \leq j \leq p$, and interior zero angles at which the boundary arcs are touching with $x^{1+\alpha}$-speed at the points $z_{j}, p+1 \leq j \leq m$. If $\alpha=0$ then the domain $G$ does not has interior zero angles and $G \in C_{\theta}(\lambda), 0<\lambda<2$, i.e., $C_{\theta}(\lambda ; 0) \equiv C_{\theta}(\lambda)$.

If $\lambda=1$ then the domain $G$ has piecewise smooth boundary with only interior zero angles. We denote the class of domains by $C_{\theta}(1 ; \alpha)$.

\subsection{New Results}

We introduce the following notation. For any $1<p<2$ and $0<\lambda<2$ we set $\alpha_{j}(p, \lambda), \beta_{j}(p, \lambda)$ and $\eta_{j}(p, \lambda)$ as follows:

$$
\alpha_{j}(p, \lambda):=\left\{\begin{array}{l}
\frac{1-\lambda}{\lambda}, \quad 0<\lambda \leq \frac{1}{2}, \quad j=1, \\
\frac{4-(p+2) \lambda}{2(2-p)+(3 p-2) \lambda}, \frac{1}{2} \leq \lambda<\frac{2}{3}, \quad j=2, \\
1+p \frac{\lambda-1}{2-\lambda}, \quad \frac{2}{3} \leq \lambda<2, \quad j=3,
\end{array}\right.
$$

Throughout this paper, $c, c_{1}, c_{2}, \cdots$ are positive and $\varepsilon, \varepsilon_{1}, \varepsilon_{2}, \cdots$, sufficiently small positive constants which in general depend on $G$.

Theorem 1.2. Let $1<p<2$ and assume that $G \in C_{\theta}(\lambda ; \alpha)$ for some $0<\lambda \leq \frac{1}{2}$ and $0 \leq \alpha<\min \left\{\alpha_{1}(p, \lambda), \beta_{1}(p, \lambda)\right\}$.

Then the $p$-Bieberbach polynomial $B_{n, p}(z)$ satisfies

$$
\left\|\varphi-B_{n, p}\right\|_{C(\bar{G})} \leq c_{1} n^{-\gamma}
$$

for each $\gamma$ with $0<\gamma<\eta_{1}-\frac{2}{p}(2+2 \alpha-p)$. 
Theorem 1.3. Let $\frac{6(2-\lambda)}{6-\lambda}<p<2$ and assume that $G \in C_{\theta}(\lambda ; \alpha)$ for some $\frac{1}{2}<\lambda \leq \frac{2}{3}$ and $0 \leq \alpha<\min \left\{\alpha_{2}(p, \lambda), \beta_{2}(p, \lambda)\right\}$. Then the $p$ - Bieberbach polynomial $B_{n, p}(z)$ satisfies

$$
\left\|\varphi-B_{n, p}\right\|_{C(\bar{G})} \leq c_{2} n^{-\gamma}
$$

for each $\gamma$ with $0<\gamma<\eta_{2}-\frac{2}{p}(2+2 \alpha-p)$.

Theorem 1.4. Let $\frac{3}{2}<p<2$ and assume that

$G \in C_{\theta}(\lambda ; \alpha)$ for some $\frac{2}{3}<\lambda<2$ and

$0 \leq \alpha<\min \left\{\alpha_{3}(p, \lambda), \frac{2 p-3}{4}\right\}$.

Then the $p$-Bieberbach polynomial $B_{n, p}(z)$ satisfies

$$
\left\|\varphi-B_{n, p}\right\|_{C(\bar{G})} \leq c_{3} n^{-\gamma}
$$

for each $\gamma$ with $0<\gamma<\eta_{3}-\frac{2}{p}(2+2 \alpha-p)$.

For $0<\lambda<1$ we obtain

$\max \left\{\alpha_{i}(p, \lambda), j=1,2,3\right\}=\alpha_{1}(p, \lambda)=\frac{1-\lambda}{\lambda}$ and so, we have the following theorem.

Theorem 1.5. Let $\max \left\{1, \frac{\left(1-4 \lambda^{2}\right)(2-\lambda)}{2 \lambda\left(\lambda^{2}-2 \lambda+2\right)}\right\}<p<2$

and assume that $G \in C_{\theta}(\lambda ; \alpha)$ for some $\frac{2}{9}<\lambda<1$ and $\frac{1-\lambda}{\lambda} \leq \alpha<\beta_{4}(p, \lambda)$. Then the $p$-Bieberbach polynomial $B_{n, p}(z)$ satisfies

$$
\left\|\varphi-B_{n, p}\right\|_{C(\bar{G})} \leq c_{4} \sqrt{|\ln n|} n^{-\gamma}
$$

for each $\gamma$ with $0<\gamma<\eta_{4}-\frac{2}{p}(2+2 \alpha-p)$.

Analogously result can be written for case $1 \leq \lambda<2$.

Theorem 1.6. Let $2<p<2+\frac{2}{|\lambda-1|}$ and assume that $G \in C_{\theta}(\lambda ; \alpha)$ for some $0<\lambda<2$, and $\alpha \geq 0$. Then, for any $n \geq 3$ and arbitrary small $\varepsilon>0$.

$$
\left\|\varphi-B_{n, p}\right\|_{C(\bar{G})} \leq c_{5} n^{-\gamma}
$$

for each $\gamma$ with $0<\gamma<\frac{1}{p}$, if $0<\lambda<1$ and $0<\gamma<\frac{1}{p}-\left(\frac{2}{p}-1\right)(\lambda-1), \quad$ if $1 \leq \lambda<2$.
Corollary 1.7. Let $p=2$ and assume that $G \in C_{\theta}(\lambda ; \alpha)$ for some $0<\lambda<2$ and $0 \leq \alpha<\frac{1}{2} \min \left\{\frac{1}{2}, \frac{\lambda}{2-\lambda}\right\}$. Then the Bieberbach polynomial $B_{n, p}(z)$ satisfies

$$
\left\|\varphi-B_{n, 2}\right\|_{C(\bar{G})} \leq c_{6} \sqrt{\log n} n^{-\gamma}
$$

for each $\gamma$ with $0<\gamma<\min \left\{\frac{1}{2}, \frac{\lambda}{2-\lambda}\right\}-2 \alpha$.

Remark 1.1. 1) Theorems $1.2-1.2$, extend the corresponding results in $[3-5,10,12,13]$ to case $p \neq 2$.

2) Corollary 1.2 is extending the one result [10] to domains bounded by a piecewise smooth curve with interior zero angles and in $\alpha=0$ coincides with the corresponding result of this work.

\section{Some Auxiliary Results}

The notation " $a \preccurlyeq b$ ", we mean that $a \leq c_{1} b$ for a constant $c_{1}$, which doesn't depend on $a$ and $b$. The relation " $a \asymp b$ " indicates that $c_{2} b \leq a \leq c_{3} b$, where $c_{2}, c_{3}$ are independent of $a$ and $b$.

Let $G \subset \mathbb{C}$ be finite domain bounded by Jordan curve $L$ and let $w=\Phi(z)(w=\hat{\varphi}(z))$ be the conformal mapping of $\Omega:=\operatorname{ext} \bar{G}(G)$ onto $\hat{\Delta}=\{w:|w|>1\} \quad(\{w:|w|<1\})$, normalized by $\Phi(\infty)=\infty, \Phi^{\prime}(\infty)>0 \quad\left(\hat{\varphi}\left(\zeta_{0}\right)=0, \hat{\varphi}^{\prime}\left(\zeta_{0}\right)>0\right)$.

The level curve (exterior or interior) can be defined for $t>0$ as

$$
L_{t}:=\{z:|\hat{\varphi}(z)|=t, \text { ift }<1 ;|\Phi(z)|=t, \text { ift }>1\}, L_{1} \equiv L .
$$

Let us denote $G_{t}:=\operatorname{int} L_{t}, \Omega_{t}:=\operatorname{ext} L_{t}$ and $d(z, L):=\inf \{|\varsigma-z|: \varsigma \in L\}$.

Let $L$ be a $K$-quasiconformal curve and $D \subset \overline{\mathbb{C}}$. Then the region $D$ can be chosen to be the region $G_{R_{0}} \backslash G_{r_{0}}$, for a certain number $1<R_{0} \leq 2$ depending on $\hat{\varphi}, \Phi, f$ and $r_{0}=R_{0}^{-1}$. In this case, it is known that the function $\alpha^{*}()=.f^{-1}\left\{[\overline{f(.)}]^{-1}\right\}$ is a $K^{2}$ - quasiconformal reflection across $L$ as shown in ([18], p. 28) by analogously in ([19], p. 75), that is, $\alpha^{*}($.$) is a K^{2}-$ quasiconformal mapping leaving points on $L$ fixed and satisfying the conditions $\alpha\left(G_{\tilde{R}} \backslash \bar{G}\right) \subset G \backslash \bar{G}_{r_{0}}$, $\alpha\left(G \backslash \bar{G}_{r}^{\tilde{r}}\right) \subset G_{R_{0}} \backslash \bar{G}$ for some $1<\tilde{R}<R_{0}, \quad r_{0}<\tilde{r}<1$. By using the facts in ([16], p. 97) and ([19], p. 76, [20], p. $26)$ we can find a $C(K)$ - quasiconformal reflection $\alpha($.) across $L$ such that it satisfies the following 


$$
\begin{gathered}
\left|z_{1}-\alpha(z)\right| \asymp\left|z_{1}-z\right|, z_{1} \in L, \varepsilon<|z|<\frac{1}{\varepsilon}, \\
\left|\alpha_{\bar{z}}\right| \asymp\left|\alpha_{z}\right| \asymp 1, \varepsilon<|z|<\frac{1}{\varepsilon}, \\
\left|\alpha_{\bar{z}}\right| \asymp\left|\alpha_{z}\right|^{2},|z|<\varepsilon,\left|\alpha_{\bar{z}}\right| \asymp|z|^{-2},|z|>\frac{1}{\varepsilon},
\end{gathered}
$$

and Jacobian $J_{\alpha}=\left|\alpha_{z}\right|^{2}-\left|\alpha_{\bar{z}}\right|^{2}$ of $\alpha($.$) satisfied$ $J_{\alpha} \asymp 1$.

Therefore, by means of the extension theorem of a quasiconformal mapping, without loss of generality we may assume that

$$
\alpha^{*}(z)=\alpha(z), z \in D .
$$

Lemma 2.1. ([18]) Let $L$ be a $K$-quasiconformal curve;

$$
\begin{gathered}
z_{1} \in L ; z_{2}, z_{3} \in G \cap\left\{z:\left|z-z_{1}\right| \leq c_{1} d\left(z_{1}, L_{R_{0}}\right)\right\}, \\
w_{j}=\hat{\varphi}\left(z_{j}\right) \\
\left(o r z_{2}, z_{3} \in G \cap\left\{z:\left|z-z_{1}\right| \leq c_{2} d\left(z_{1}, L_{\rho_{0}}\right)\right\}, w_{j}=\Phi\left(z_{j}\right)\right) \\
j=1,2,3 .
\end{gathered}
$$

Then,

1) The statements $\left|z_{1}-z_{2}\right| \preccurlyeq\left|z_{1}-z_{3}\right|$ and $\left|w_{1}-w_{2}\right| \preccurlyeq\left|w_{1}-w_{3}\right|$ are equivalent. So are $\left|z_{1}-z_{2}\right| \asymp\left|z_{1}-z_{3}\right|$ and $\left|w_{1}-w_{2}\right| \asymp\left|w_{1}-w_{3}\right|$

2) If $\left|z_{1}-z_{2}\right| \preccurlyeq\left|z_{1}-z_{3}\right|$, then

$$
\left|\frac{w_{1}-w_{3}}{w_{1}-w_{2}}\right|^{K^{-2}} \preccurlyeq\left|\frac{z_{1}-z_{3}}{z_{1}-z_{2}}\right| \preccurlyeq\left|\frac{w_{1}-w_{3}}{w_{1}-w_{2}}\right|^{K^{2}}
$$

and, consequently, for any $z_{3} \in L_{R_{0}}\left(z_{3} \in L_{\rho_{0}}\right)$

$$
\left|w_{1}-w_{2}\right|^{K^{2}} \preccurlyeq\left|z_{1}-z_{2}\right| \preccurlyeq\left|w_{1}-w_{2}\right|^{K^{-2}},
$$

where $1<R_{0}<2$ and $\rho_{0}=R_{0}^{-1}$ are fixed constants.

Lemma 2.2. ([21]) Let $L:=\partial G$ be a quasiconformal curve. Then, For every $z \in L$ there exists an arc $\beta\left(\zeta_{0}, z\right)$ in $G$ joining $\zeta_{0}$ to $z$ with following properties:

1) $d(\xi, L) \asymp|\xi-z|$ for every $\xi \in \beta\left(\zeta_{0}, z\right)$

2) If $\tilde{\beta}\left(\xi_{1}, \xi_{2}\right)$ is the subarc of $\beta\left(\zeta_{0}, z\right)$ joining $\xi_{1}$ to $\xi_{2}$, then mes $\tilde{\beta}\left(\xi_{1}, \xi_{2}\right) \preccurlyeq\left|\xi_{1}-\xi_{2}\right|$ for every pair $\xi_{1}, \xi_{2} \in \beta\left(\zeta_{0}, z\right)$.

Lemma 2.3. ([7]) Let $L:=\partial G$ be a $K$ - quasiconformal curve. Then mes $\ell \asymp$ mes $\alpha(\ell)$, for every rectifiable arc $\ell \subset G$.

Let $G$ be arbitrary Jordan domain and $\Gamma:=\Gamma\left(z_{0}, \xi\right) \in \Omega, z_{0} \in L, \xi \in \Omega$ an rectifiable arc except for one of its endpoints of its $z_{0} \in L$ which satisfies the following conditions:

1) mes $\Gamma\left(\xi_{1}, \xi_{2}\right) \preccurlyeq\left|\xi_{1}-\xi_{2}\right|$, for all $\xi_{1}, \xi_{2} \in \Gamma$.

2) There exists a monotone increasing function $g(t)$ such that $d(\xi, L) \succcurlyeq g\left(\left|\xi-z_{0}\right|\right)$ for all $\xi \in \Gamma$.

Lemma 2.4. ([22]) Let $G \in C_{\theta}(\lambda ; \alpha)$ for some $0<\lambda<2, \quad \alpha \geq 0$ and on the arc $\Gamma$ a measurable function $f(\xi)$ be given such that $|f(\xi)| \preccurlyeq\left|\xi-z_{0}\right|^{\frac{1}{2-\lambda}}$ for all $\xi \in \Gamma$. Then for all $z \notin \Gamma$.

$$
\left\|\int_{\Gamma} \frac{f(\xi)}{(\xi-z)^{2}} \mathrm{~d} \xi\right\|_{A_{p}(G)} \preccurlyeq \begin{cases}|\ln \ell|^{\frac{1}{2} \ell^{\frac{2}{p}+\frac{\lambda-1}{2-\lambda}}} & 1<p<2, \\ \ell^{\frac{2}{p}+\frac{\lambda-1}{2-\lambda}} & 2 \leq p<2+\frac{2}{|\lambda-1|} .\end{cases}
$$

\section{Approximation in the $A_{p}^{1}(G)$-Norm}

Suppose that $G \in C_{\theta}(\lambda ; \alpha)$ for some $0<\lambda<2$ and $\alpha \geq 0$ is given. For the sake of simplicity, but without loss of generality, we assume that $m=2, z_{1}=1$, $z_{2}=-1 ; \quad(-1,1) \subset G$ and let the local coordinate axes be parallel to $O X$ and $O Y$ in the coordinate system; $L^{1}:=\{z: z \in L, \operatorname{Im} z \geq 0\}, L^{2}:=\{z: z \in L, \operatorname{Im} z \leq 0\}$. Then $z_{0}$ is taken as an arbitrary point on $L^{2}$ (or on $L^{1}$ subject to the chosen direction).

We recall that the domain $G \in C_{\theta}(\lambda ; \alpha)$ has exterior $\lambda \pi \quad(0<\lambda<2)$ non zero angle and $x^{1+\alpha}$ type interior zero angle in the neighborhood of the points $z_{1}=-1$ and $z_{2}=1$, respectively.

We can say that the function $w=\hat{\varphi}(z)$ for the domain $G \in C_{\theta}(\lambda ; \alpha)$ satisfies the conditions described in Lemma 2 in the neighborhood of point $z_{1}=-1$. So, we can easily get from Lemma 2

$$
d(z, L) \preccurlyeq(|\hat{\varphi}(z)|-1)^{(1+\varepsilon)^{-2}} ;|z-1| \preccurlyeq|\hat{\varphi}(z)-\hat{\varphi}(1)|^{(1+\varepsilon)^{-2}},
$$

for all $z \in M:=\left\{z \in G:|z+1|>\varepsilon_{1}\right\}$.

On the other hand, using properties of the function $w=\hat{\varphi}(z)$ in the neigbourhood of the point $z_{1}=-1$ (see, $[7,23])$ we obtain

$$
|z+1| \leq[-\ln |\hat{\varphi}(z)-\hat{\varphi}(-1)|]^{-\alpha^{-1}}
$$

Because each $L^{j}, j=1,2$ is a $\left(1+\varepsilon_{i}\right)$ - quasiconformal arc, $\alpha_{j}($.$) must be the quasiconformal$ reflection across $L^{j}$. Let us consider the curve

$$
\begin{gathered}
\gamma_{1}^{1}:=\alpha_{1}\left\{z=x+i y: y=\frac{2 \tilde{c}_{1}+\tilde{c}_{2}}{3}(x+1)^{1+\alpha}\right\} ; \\
\gamma_{1}^{2}:=\alpha_{2}\left\{z=x+i y: y=\frac{\tilde{c}_{1}+2 \tilde{c}_{2}}{3}(x+1)^{1+\alpha}\right\} ; \\
\gamma_{2}^{1}:=z=x+i y: y=\tilde{c}_{3}(x-1) ; \\
\gamma_{2}^{2}:=z=x+i y: y=\tilde{c}_{4}(x-1) ;
\end{gathered}
$$


for some $-\infty<\tilde{c}_{4}<\tilde{c}_{3}<\infty$, where a constants $\tilde{c}_{1}$ and $\tilde{c}_{2}$ from Definition 1.3.

It is easy to check from Lemma 2.2 that

$$
\text { mes } \gamma_{j}^{i}\left(\xi_{1}, \xi_{2}\right) \preccurlyeq\left|\xi_{1}-\xi_{2}\right| \text {, }
$$

for all $\xi_{1}, \xi_{2} \in \gamma_{j}^{i}, i, j=1,2$.

Let $N=N\left(R_{0}\right)$ sufficiently large natural number. For $n>N$ and arbitrary $0<\varepsilon<1$, let us choose $R=r_{0}+c n^{\varepsilon-1}$ such that $r_{0}<R<R_{0}$ and points $z_{j}^{i}, i, j=1,2$ such that they are in the intersection of $L_{R}$ and $\gamma_{j}^{i}$

These points divide $L_{R}$ into four parts:

$$
\begin{aligned}
& L_{R}^{1}:=L_{R}\left(z_{2}^{1}, z_{1}^{1}\right), L_{R}^{3}:=L_{R}\left(z_{1}^{1}, z_{1}^{2}\right), \\
& L_{R}^{2}:=L_{R}\left(z_{1}^{2}, z_{2}^{2}\right), L_{R}^{4}:=L_{R}\left(z_{2}^{2}, z_{2}^{1}\right)
\end{aligned}
$$

are traversed in the positive direction (counterclockwise). $L_{R}:=L_{R}^{1} \cup L_{R}^{3} \cup L_{R}^{2} \cup L_{R}^{4}$ and $\gamma_{j}^{i}(R)$ are subarcs of $\gamma_{j}^{i}$ joining points $-1,1$ with $z_{j}^{i}$. Let us denote

$\Gamma_{R}:=\gamma_{1}^{1}(R) \cup L_{R}^{1} \cup \gamma_{2}^{1}(R) \cup \gamma_{2}^{2}(R) \cup L_{R}^{2} \cup \gamma_{1}^{2}(R)$, $U:=\operatorname{int}\left(\Gamma_{R}\right)$ and $U_{R}:=U \backslash \bar{G}$.

We can extend the function $\varphi(z)$ to $U$ in the following way

$$
\tilde{\varphi}(z):= \begin{cases}\varphi(z), & z \in \bar{G}, \\ \frac{r_{0}^{2}}{\overline{\varphi\left(\alpha_{i}(z)\right)}}, & z \in U_{R}, i=1,2 .\end{cases}
$$

Then,

$$
\tilde{\varphi}_{\bar{z}}(z)= \begin{cases}0, & z \in G, \\ \varphi^{\prime}\left(\alpha_{i}(z)\right) \alpha_{i, \bar{z}}(z), & z \in U_{R}, i=1,2 .\end{cases}
$$

From the Cauchy-Pompeiu formula ([16], p. 148), we get

$$
\varphi(z)=\frac{1}{2 \pi i} \int_{\Gamma_{R}} \frac{\tilde{\varphi}(\xi)}{\xi-z} \mathrm{~d} \xi-\frac{1}{\pi} \int_{U_{R}} \frac{\tilde{\varphi}_{\bar{\xi}}(\xi)}{\xi-z} \mathrm{~d} \sigma_{\xi}, z \in G .
$$

Then, using the above notations we obtain

$$
\begin{aligned}
\varphi(z) & =\frac{1}{2 \pi i} \int_{L_{R}} \frac{f(\xi)}{\xi-z} \mathrm{~d} \xi \\
& +\frac{1}{2 \pi i} \sum_{i, j=1}^{2} \int_{\gamma_{j}(R)} \frac{\tilde{\varphi}(\xi)-\varphi\left((-1)^{j}\right)}{\xi-z} \mathrm{~d} \xi \\
& -\frac{1}{\pi} \iint_{U_{R}} \frac{\tilde{\varphi}_{\bar{\xi}}(\xi)}{\xi-z} \mathrm{~d} \sigma_{\xi},
\end{aligned}
$$

$$
f(\xi)= \begin{cases}\tilde{\varphi}(\xi) & \xi \in L_{R}^{1} \text { or } \xi \in L_{R}^{2} \\ \varphi(-1) & \xi \in L_{R}^{3} \\ \varphi(1) & \xi \in L_{R}^{4} .\end{cases}
$$

Since the first part of right hand side in (3.5) is analytic function in $\bar{G}$, there exists a polynomial $P_{n-1}(z)$, where $\operatorname{deg} P_{n-1}(z) \leq n-1 \quad$ ([24], p. 142), such that

$$
\left|\frac{1}{2 \pi i} \int_{L_{R}} \frac{f(\xi)}{(\xi-z)^{2}} \mathrm{~d} \xi-P_{n-1}(z)\right|<\frac{c}{n} .
$$

Let $Q_{n}(z):=\int_{\zeta_{0}}^{z} P_{n-1}(t) \mathrm{d} t$. Then, $Q_{n}\left(\zeta_{0}\right)=0$ and from (3.5) and (3.6) we have

$$
\begin{aligned}
\left|\varphi^{\prime}(z)-Q_{n}^{\prime}(z)\right| \leq & \frac{c}{n}+\frac{1}{2 \pi}\left|\sum_{i, j=1}^{2} \int_{\gamma_{j}^{i}(R)} \frac{\tilde{\varphi}(\xi)-\varphi\left((-1)^{j}\right)}{(\xi-z)^{2}} \mathrm{~d} \xi\right| \\
& +\left|-\frac{1}{\pi} \iint_{U_{R}} \frac{\tilde{\varphi}_{\bar{\xi}}(\xi)}{(\xi-z)^{2}} \mathrm{~d} \sigma_{\xi}\right|
\end{aligned}
$$

and let us take integrals over $G$ of the p-th power of each side we obtain

$$
\begin{aligned}
& \iint_{G}\left|\varphi^{\prime}(z)-Q_{n}^{\prime}(z)\right|^{p} \mathrm{~d} \sigma_{z} \\
& \preccurlyeq \frac{1}{n^{p}}+\iint_{G}\left|\sum_{i, j=1}^{2} \int_{\gamma_{j}^{\prime}(R)} \frac{\tilde{\varphi}(\xi)-\varphi\left((-1)^{j}\right)}{(\xi-z)^{2}} \mathrm{~d} \xi\right|^{p} \mathrm{~d} \sigma_{z} \\
& \quad+\left.\iint_{G} \iint_{U_{R}} \frac{\tilde{\varphi}_{\bar{\xi}}(\xi)}{(\xi-z)^{2}} \mathrm{~d} \sigma_{\xi}\right|^{p} \mathrm{~d} \sigma_{z} .
\end{aligned}
$$

From the Calderon-Zygmund inequality ([19], p. 98), we obtain

$$
\iint_{G}\left|\int_{U_{R}} \frac{\tilde{\varphi}_{\bar{\xi}}(\xi)}{(\xi-z)^{2}} \mathrm{~d} \sigma_{\xi}\right|^{p} \mathrm{~d} \sigma_{z} \preccurlyeq \iint_{U_{R}}\left|\varphi^{\prime}\left(\alpha_{i}(\xi)\right)\right|^{p} \mathrm{~d} \sigma_{\xi}, i=1,2 .
$$

So, (3.7) and (3.8) give us

$$
\begin{aligned}
\left\|\varphi^{\prime}-Q_{n}^{\prime}\right\|_{A_{p}(G)}^{p} \preccurlyeq & \frac{1}{n^{p}}+\sum_{i, j=1}^{2}\left\|\int_{\gamma_{j}(R)} \frac{\tilde{\varphi}(\xi)-\varphi\left((-1)^{j}\right)}{(\xi-z)^{2}} \mathrm{~d} \xi\right\|_{A_{p}(G)}^{p} \\
& +\iint_{U_{R}}\left|\varphi^{\prime}\left(\alpha_{i}(\xi)\right)\right|^{p} \mathrm{~d} \sigma_{\xi} .
\end{aligned}
$$

where 
Let us consider two case of $p$ in the last double integral in (3.9) as: $1<p<2$ and $p \geq 2$.
If $1<p<2$, then, using Hölder Inequality ([25], p. 105) we obtain

$$
\begin{aligned}
\iint_{U_{R}}\left|\varphi^{\prime}\left(\alpha_{i}(\xi)\right)\right|^{p} \mathrm{~d} \sigma_{\xi} & \preccurlyeq\left(\iint_{U_{R}}\left|\varphi^{\prime}\left(\alpha_{i}(\xi)\right)\right|^{2} \mathrm{~d} \sigma_{\xi}\right)^{\frac{p}{2}}\left(\iint_{U_{R}} \mathrm{~d} \sigma_{\xi}\right)^{1-\frac{p}{2}} \\
& \preccurlyeq\left(\iint_{\alpha_{i}\left(U_{R}\right)}\left|\varphi^{\prime}(\xi)\right|^{2} \mathrm{~d} \sigma_{\xi}\right)^{\frac{p}{2}}\left(\iint_{\alpha_{i}\left(U_{R}\right)} \mathrm{d} \sigma_{\xi}\right)^{1-\frac{p}{2}} \\
& \preccurlyeq\left(\operatorname{mes} \varphi\left(\alpha_{i}\left(U_{R}\right)\right)\right)^{\frac{p}{2}}\left(\operatorname{mes}\left(\alpha_{i}\left(U_{R}\right)\right)\right)^{1-\frac{p}{2}} .
\end{aligned}
$$

Thus, (3.9) and (3.10) give us

$$
\begin{aligned}
\left\|\varphi^{\prime}-Q_{n}^{\prime}\right\|_{A_{p}(G)}^{p} \preccurlyeq & \frac{1}{n^{p}}+\sum_{i, j=1}^{2}\left\|\int_{\gamma_{j}^{i}(R)} \frac{\tilde{\varphi}(\xi)-\varphi\left((-1)^{j}\right)}{(\xi-z)^{2}} \mathrm{~d} \xi\right\|_{A_{p}(G)}^{p} \\
& + \begin{cases}\left(\operatorname{mes} \varphi\left(\alpha_{i}\left(U_{R}\right)\right)\right)^{\frac{p}{2}}\left(\operatorname{mes}\left(\alpha_{i}\left(U_{R}\right)\right)\right)^{1-\frac{p}{2}}, & 1<p<2, i=1,2, \\
\iint_{U_{R}}\left|\varphi^{\prime}\left(\alpha_{i}(\xi)\right)\right|^{p} d \sigma_{\xi}, & p \geq 2, i=1,2 .\end{cases}
\end{aligned}
$$

Now, we need some technical estimates to attack the problem in (3.11).
Lemma 3.1. Let $G \in C_{\theta}(\lambda ; \alpha)$ for some $0<\lambda<2$, $\alpha \geq 0$. Then for all $\varepsilon>0$

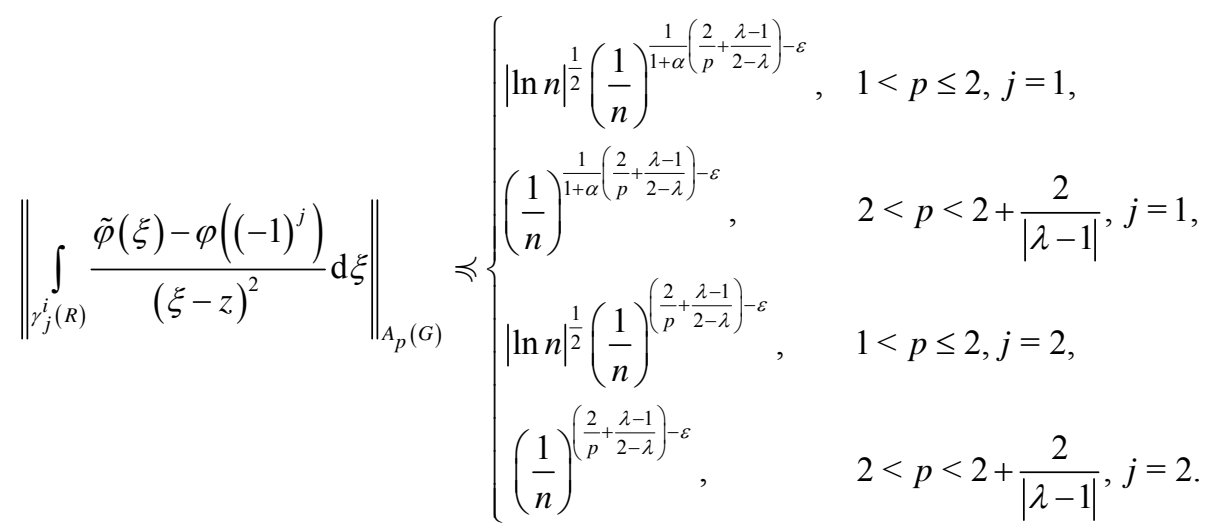

Proof. Let us choose $f(\xi):=\tilde{\varphi}(\xi)-\varphi\left((-1)^{j}\right), \quad j=1,2$, in Lemma 2.4, we obtain

$$
\left\|\int_{\gamma_{j}^{i}(R)} \frac{\tilde{\varphi}(\xi)-\varphi\left((-1)^{j}\right)}{(\xi-z)^{2}} \mathrm{~d} \xi\right\|_{A_{p}(G)} \preccurlyeq\left\{\begin{array}{lc}
\left|\ln \ell_{i, j}\right|^{\frac{1}{2}} \ell_{i, j}^{\frac{2}{p}+\frac{\lambda-1}{2-\lambda}}, & 1<p \leq 2, \\
\ell_{i, j}^{\frac{2}{p}+\frac{\lambda-1}{2-\lambda}}, & 2<p<2+\frac{2}{|\lambda-1|},
\end{array}\right.
$$

where $\ell_{i, j}:=\operatorname{mes} \gamma_{j}^{i}(R)$. On the other hand, according to Lemma 2.1 and Corollary 1.1, we have $d\left(z_{j}^{i}, L^{i}\right) \preccurlyeq n^{\varepsilon-1}$. Then, from (2.1), (3.1) and (3.2), for all $\varepsilon>0$, we get 


$$
\ell_{i, j} \preccurlyeq\left|z_{j}^{i}-(-1)^{j}\right| \preccurlyeq\left\{\begin{array} { c c } 
{ d ^ { \frac { 1 } { 1 + \alpha } } ( z _ { j } ^ { i } , L ^ { i } ) , } & { j = 1 } \\
{ d ( z _ { j } ^ { i } , L ^ { i } ) , } & { j = 2 }
\end{array} \preccurlyeq \left\{\begin{array}{cc}
\left(\frac{1}{n}\right)^{\frac{1}{1+\alpha}-\varepsilon}, & j=1 \\
\left(\frac{1}{n}\right)^{1-\varepsilon}, & j=2
\end{array} .\right.\right.
$$

Then, the following inequalities are obtained from

(3.12) and (3.13)

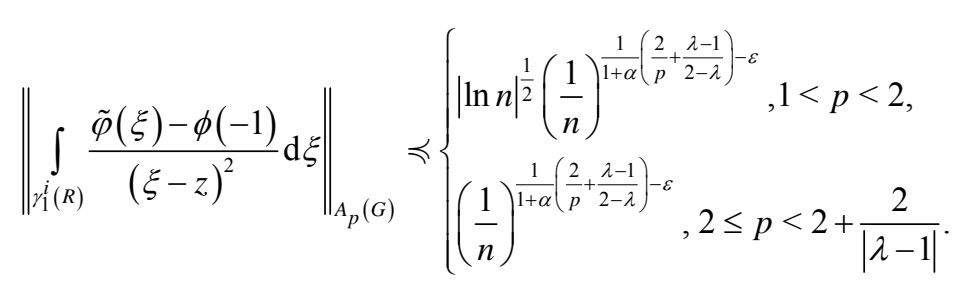

and

$$
\left\|\int_{\gamma_{2}^{i}(R)} \frac{\tilde{\varphi}(\xi)-\varphi(1)}{(\xi-z)^{2}} \mathrm{~d} \xi\right\|_{A_{p}(G)} \preccurlyeq\left\{\begin{array}{l}
|\ln n|^{\frac{1}{2}\left(\frac{1}{n}\right)^{\left(\frac{2}{p}+\frac{\lambda-1}{2-\lambda}\right)-\varepsilon}, 1<p<2,} \\
\left(\frac{1}{n}\right)^{\left(\frac{2}{p}+\frac{\lambda-1}{2-\lambda}\right)-\varepsilon}, 2 \leq p<2+\frac{2}{|\lambda-1|} .
\end{array}\right.
$$

Combining (3.14) and (3.15) the proof is completed.

Now, for sufficiently small $0<\varepsilon_{0}<\varepsilon_{1}$, we are going to use following notations:

$$
\begin{aligned}
U_{R} & :=V_{1}^{1} \cup V_{1}^{2} \cup V_{2}^{1} \cup V_{2}^{2} \cup V_{3}^{1} \cup V_{3}^{2} \text { where } \\
V_{j}^{1} & :=U_{R} \cap D\left((-1)^{j}, \varepsilon_{0}\right) \cap\{z: \operatorname{Imz}>0\}, j=1,2 \\
V_{j}^{2} & :=U_{R} \cap D\left((-1)^{j}, \varepsilon_{0}\right) \cap\{z: \operatorname{Imz}<0\}, j=1,2 \\
V_{3}^{1}: & =\left[U_{R} \cap\{z: \operatorname{Imz}>0\}\right] \backslash\left[D\left(-1, \varepsilon_{0}\right) \cup D\left(1, \varepsilon_{0}\right)\right], \\
V_{3}^{2} & :=\left[U_{R} \cap\{z: \operatorname{Imz}<0\}\right] \backslash\left[D\left(-1, \varepsilon_{0}\right) \cup D\left(1, \varepsilon_{0}\right)\right] .
\end{aligned}
$$

Lemma 3.2. ([26], p. 10) Let $G \in C_{\theta}(\lambda ; \alpha)$ for some $0<\lambda<2, \quad \alpha \geq 0$. Then for all $\varepsilon>0$

$$
\operatorname{mes} \varphi\left(\alpha_{i}\left(V_{1}^{i}\right)\right) \preccurlyeq\left(\frac{1}{n}\right)^{1-\varepsilon}, \operatorname{mes} \varphi\left(\alpha_{i}\left(V_{3}^{i}\right)\right) \preccurlyeq\left(\frac{1}{n}\right)^{1-\varepsilon} \text {, }
$$

and

$$
\operatorname{mes} \varphi\left(\alpha_{i}\left(V_{2}^{i}\right)\right) \preccurlyeq\left(\frac{1}{n}\right)^{2 \mu-\varepsilon}, i=1,2
$$

where $\mu:=\min \left\{\frac{\lambda}{2-\lambda}, \frac{1}{2}\right\}$.

Lemma 3.3. Let $G \in C_{\theta}(\lambda ; \alpha)$ for some $0<\lambda<2$, $\alpha \geq 0$. Then for all $\varepsilon>0$

$$
\text { mes } \alpha_{i}\left(U_{R}\right) \preccurlyeq\left(\frac{1}{n}\right)^{\min \{2 \lambda, 1\}-\varepsilon}, i=1,2 \text {. }
$$

Proof. We have

$$
\alpha_{i}\left(U_{R}\right)=\sum_{i=1}^{2}\left[\operatorname{mes} \alpha_{i}\left(V_{1}^{i}\right)+\operatorname{mes}\left(V_{2}^{i} \cup V_{3}^{i}\right)\right]
$$

Now, let us estimate mes $\alpha_{i}\left(V_{1}^{i}\right)$ by choosing $K=1+\varepsilon, \varepsilon>0$ and from Lemma 2.1 we have

$$
\begin{aligned}
& \text { mes } \alpha_{i}\left(V_{3}^{i}\right) \preccurlyeq\left[d\left(z_{1}^{i}, L^{i}\right)\right]^{\frac{1}{(1+\varepsilon)^{2}}} \preccurlyeq\left(\frac{1}{n}\right)^{1-\varepsilon} . \\
& \operatorname{mes} \alpha_{i}\left(V_{1}^{i}\right) \preccurlyeq\left[d\left(z_{1}^{i}, L^{i}\right)\right]^{\frac{1}{(1+\varepsilon)^{2}}} \preccurlyeq\left(\frac{1}{n}\right)^{1-\varepsilon}
\end{aligned}
$$

On the other hand if we use the method of Gaier in [9] but except the conformal mapping $\varphi$ in method, then we obtain

$$
\operatorname{mes}\left(V_{2}^{i}\right) \preccurlyeq\left(\frac{1}{n}\right)^{\min \{2 \lambda, 1\}-\varepsilon} .
$$

After then using the second and third inequality in the first one we obtain the desired proof.

Lemma 3.4. Let $G \in C_{\theta}(\lambda ; \alpha)$ for some $0<\lambda<2$, $\alpha \geq 0$. Then for all $\varepsilon>0$

$$
\iint_{U_{R}}\left|\varphi^{\prime}\left(\alpha_{i}(\xi)\right)\right|^{p} \mathrm{~d} \sigma_{\xi} \preccurlyeq\left(\frac{1}{n}\right)^{1-(2-p)\left(1-\theta_{*}\right)-\varepsilon}, i=1,2
$$

where $2 \leq p<2+\frac{1}{1-\theta_{*}}$ and $\theta_{*}=\min \{\lambda, 2-\lambda\}$.

Proof. Let $z \in \bar{G}, \quad Z^{*}-$ the point of $L:=\partial G$ nearest to $z$. Then we have

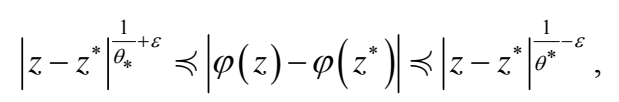

and from ([18], Lemma 3) 


$$
\left|\psi^{\prime}(w)\right| \asymp \frac{d(\psi(w), L)}{r_{o}-|w|} \asymp \frac{\left|\psi(w)-\psi\left(w^{*}\right)\right|}{r_{o}-|w|} \asymp \frac{\left|w-w^{*}\right|^{\theta_{*}-\varepsilon}}{r_{0}-|w|} \asymp\left(\frac{1}{r_{0}-|w|}\right)^{1-\theta_{*}+\varepsilon} .
$$

After this estimation, we get

$$
\begin{aligned}
\iint_{U_{R}}\left|\varphi^{\prime}\left(\alpha_{i}(\xi)\right)\right|^{p} \mathrm{~d} \sigma_{\xi} & \preccurlyeq \iint_{\alpha_{i}\left(U_{R}\right)}\left|\varphi^{\prime}(\xi)\right|^{p} \mathrm{~d} \sigma_{\xi} \asymp \iint_{\varphi\left(\alpha_{i}\left(U_{R}\right)\right)}\left(\frac{1}{\left|\psi^{\prime}(w)\right|}\right)^{(p-2)} \mathrm{d} \sigma_{w} \\
& \asymp \iint_{\varphi\left(\alpha_{i}\left(U_{R}\right)\right)}\left(r_{0}-|w|\right)^{(p-2)\left(\theta_{*}-1\right)} \mathrm{d} \sigma_{w} \asymp\left(r_{0}-r_{*}\right)^{1-(p-2)\left(1-\theta_{*}\right)-\varepsilon} \asymp\left(\frac{1}{n}\right)^{1-(p-2)\left(1-\theta_{*}\right)-\varepsilon},
\end{aligned}
$$

where $r_{*}:=\min \left\{\left|\varphi\left(\alpha_{i}(z)\right)\right|: z \in L_{R}\right\}$ and $2 \leq p<2+\frac{1}{1-\theta_{*}}$.
By using Lemma 3.1, 3.2, 3.3, 3.4 and (3.11) we get the following results. We need this notation:

$$
\vartheta_{n}(p, \lambda):=\left\{\begin{array}{lll}
|\ln n|^{\frac{1}{2}}\left(\frac{1}{n}\right)^{\frac{1}{1+\alpha}\left(\frac{2}{p}+\frac{\lambda-1}{2-\lambda}\right)-\varepsilon}, & \alpha \geq \max \left\{\alpha_{i}(p, \lambda)\right\}, & i=1,2,3 . \\
\left(\frac{1}{n}\right)^{\frac{\lambda}{2-\lambda}+\frac{2 \lambda}{p}-\lambda-\varepsilon}, & \alpha<\alpha_{1}(p, \lambda), & 0<\lambda \leq \frac{1}{2} . \\
\left(\frac{1}{n}\right)^{\frac{\lambda}{2-\lambda}+\frac{1}{p}-\frac{1}{2}-\varepsilon}, & \alpha<\alpha_{2}(p, \lambda), & \frac{1}{2}<\lambda \leq \frac{2}{3} . \\
\left(\frac{1}{n}\right)^{\frac{1}{p}-\varepsilon}, & \alpha<\alpha_{3}(p, \lambda), & \frac{2}{3}<\lambda<2 .
\end{array}\right.
$$

Lemma 3.5. Let $1<p \leq 2$ and assume that $G \in C_{\theta}(\lambda ; \alpha)$ for some $0<\lambda<2, \alpha \geq 0$. Then, for any $n \geq 3$ and arbitrary small $\varepsilon>0$

$$
\left\|\varphi-B_{n, p}\right\|_{A_{p}^{1}(G)} \preccurlyeq \vartheta_{n}(p, \lambda) .
$$

Lemma 3.6. Let $2<p<2+\frac{2}{|\lambda-1|}$ and assume that $G \in C_{\theta}(\lambda ; \alpha)$ for some $0<\lambda<2, \alpha \geq 0$. Then, for any $n \geq 3$ and arbitrary small $\varepsilon>0$

$$
\left\|\varphi-B_{n, p}\right\|_{A_{p}^{1}(G)} \preccurlyeq\left\{\begin{array}{cc}
\left(\frac{1}{n}\right)^{\frac{1}{p}-\varepsilon}, & 0<\lambda<1, \\
\left(\frac{1}{n}\right)^{\frac{1-(2-p)(\lambda-1)}{p}-\varepsilon} & 1 \leq \lambda<2 .
\end{array} .\right.
$$

Proof. The proof of Lemma 3.5-3.6 is similar. So, we give them together. From (3.11) and Lemma 3.1-3.4 we obtain

$$
\begin{aligned}
& \left\|\varphi^{\prime}-Q_{n}^{\prime}\right\|_{A_{p}(G)} \preccurlyeq \frac{1}{n}+\left\{\begin{array}{cc}
|\ln n|^{\frac{1}{2}}\left(\frac{1}{n}\right)^{\frac{1}{1+\alpha}\left(\frac{2}{p}+\frac{\lambda-1}{2-\lambda}\right)-\varepsilon} & 1<p<2, \quad j=1, \\
\left(\frac{1}{n}\right)^{\frac{1}{1+\alpha}\left(\frac{2}{p}+\frac{\lambda-1}{2-\lambda}\right)-\varepsilon} & 2 \leq p<2+\frac{2}{|\lambda-1|}, \quad j=1,
\end{array}\right.
\end{aligned}
$$

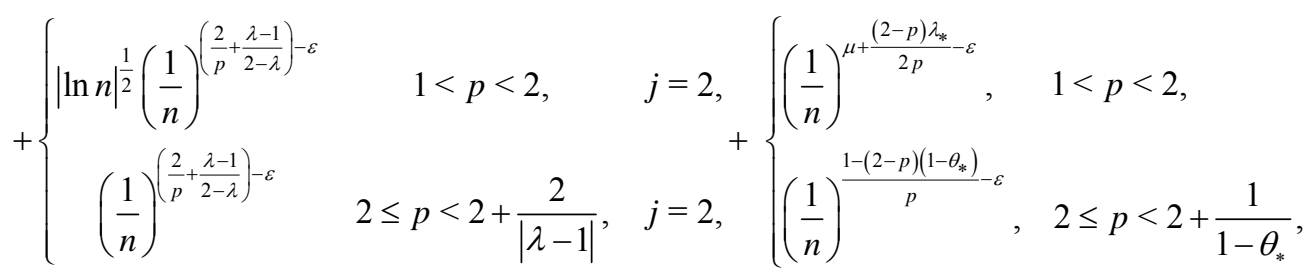


where $\mu:=\min \left\{\frac{\lambda}{2-\lambda}, \frac{1}{2}\right\}$,

$\lambda_{*}:=\min \{2 \lambda ; 1\}, \theta_{*}:=\min \{1,2-\lambda\}$

Case 1. Let $1<p<2$. In this case from (3.18) we get

$$
\begin{aligned}
\left\|\varphi^{\prime}-Q_{n}^{\prime}\right\|_{A_{p}(G)} & \preccurlyeq|\ln n|^{\frac{1}{2}}\left(\frac{1}{n}\right)^{\frac{1}{1+\alpha}\left(\frac{2}{p}+\frac{\lambda-1}{2-\lambda}\right)-\varepsilon}+\left(\frac{1}{n}\right)^{\mu+\frac{(2-p) \lambda_{*}}{2 p}-\varepsilon} \\
& \preccurlyeq \vartheta_{n}(p, \lambda) .
\end{aligned}
$$

Case 2. Let

$2 \leq p<\min \left\{2+\frac{2}{|\lambda-1|} ; 2+\frac{1}{1-\theta_{*}}\right\}, \theta_{*}:=\min \{1,2-\lambda\}$.

In this case from (3.18) we have

$$
\left\|\varphi-\tilde{Q}_{n}\right\|_{A_{p}^{1}(G)} \preccurlyeq\left\{\begin{array}{l}
\left(\frac{1}{n}\right)^{\vartheta_{\lambda}-\varepsilon}, \\
\left(\frac{1}{n}\right)^{\frac{1}{p}-\varepsilon}, \\
\left(\frac{1}{n}\right)^{\frac{1-(2-p)(\lambda-1)}{p}-\varepsilon},
\end{array}\right.
$$

So, if we consider the extremal property of the polynomials $B_{n, p} \in \wp_{n}$, then we obtain (3.16) and (3.17) respectively.

To prove Theorem 1.2-1.6 and Corollary 1.7, we use a similar method to the one of Andrievskii and Simonenko employed in the proofs of analogous theorems for $p=2$ (see $[6,8,10])$.

Lemma 3.7. [2] Let $G \subset \mathbb{C}$ be a simply connected domain so that

$$
\left\|\varphi-B_{n, p}\right\|_{A_{p}^{1}(G)} \preccurlyeq n^{-\mu}
$$

for each $\mu \in(0,1), \quad n=2,3, \cdots$, and

$$
\left\|P_{n}\right\|_{C(\bar{G})} \preccurlyeq\left\|P_{n}\right\|_{A_{p}^{1}(G)} \begin{cases}1, & p>2, \\ \sqrt{\log n}, & p=2, \\ n^{\eta}, & 0<p<2, \eta>0\end{cases}
$$

for all polynomials $P_{n}(z)$ of $\operatorname{deg} P_{n} \leq n$ and normalized with $P_{n}\left(\zeta_{0}\right)=0$. Then,

$$
\left\|\varphi-B_{n, p}\right\|_{C(\bar{G})} \preccurlyeq n^{\eta-\mu} .
$$

It is enough replacing $\mu$ by $\vartheta$ in (3.16) and (3.17) respectively and $\eta=\frac{2}{p}(2 \alpha+2-p)$ in ([22], Corollary

$$
\begin{aligned}
\left\|\varphi^{\prime}-Q_{n}^{\prime}\right\|_{A_{p}(G)} & \preccurlyeq\left(\frac{1}{n}\right)^{\frac{2}{p}+\frac{\lambda-1}{2-\lambda}-\varepsilon}+\left(\frac{1}{n}\right)^{\frac{1-(2-p)\left(1-\theta_{*}\right)}{p}-\varepsilon} \\
& \preccurlyeq \begin{cases}\left(\frac{1}{n}\right)^{\frac{1}{p}-\varepsilon}, & 0<\lambda<1, \\
\left(\frac{1}{n}\right)^{\frac{1-(2-p)(\lambda-1)}{p}-\varepsilon}, & 1 \leq \lambda<2 .\end{cases}
\end{aligned}
$$

Now, let us consider the polynomial

$\tilde{Q}_{n}(z):=Q_{n}(z)+\left[1-Q_{n}^{\prime}\left(\zeta_{0}\right)\right]\left(z-\zeta_{0}\right)$. It is clear $\tilde{Q}_{n} \in \wp_{n}$ that $\tilde{Q}_{n}\left(z_{0}\right)=0, \tilde{Q}_{n}^{\prime}\left(z_{0}\right)=1$. So, we have

$1<p<2$,

$0<\lambda<1,2 \leq p<2+\frac{2}{|\lambda-1|}$,

$1 \leq \lambda<2,2 \leq p<2+\frac{2}{|\lambda-1|}$.

4.1) in (3.19) to prove Theorems 1.2-1.6.

\section{References}

[1] P. J. Davis, "Interpolation and Approximation," Blaisdell Pub. Company, New York, 1963.

[2] M. Kucukaslan and F. G. Abdullayev, "New Extremal Polynomials and Its Approximations Properties," Novisad Journal of Mathematics, Vol. 39, No. 2, 2009, pp. 1-12.

[3] M. V. Keldysh, "Sur L'approximation en Moyenne Quadratique des Fontions Analtiques," Matematicheskii Sbornik, Vol. 5, No. 2, 1939, pp. 391-401.

[4] P. K. Suetin, "Polynomials Orthogonal over a Region and Bieberbach Polynomial," American Mathematical Society, Rohde Island, 1974.

[5] S. N. Mergelyan, "Certain Questionsof the Constructive Theory of Functions," Trudy Matematicheskogo Instituta im. V.A. Steklova RAN, Vol. 37, 1951, pp. 3-91.

[6] I. B. Simonenko, "On the Convergence of Bieberbach Polynomials in the Case of Lipschitz Domain," Mathematics of the USSR-Izvestiya, Vol. 13, No. 1, 1979, pp. 166-174. doi:10.1070/IM1979v013n01ABEH002017

[7] V. V. Andrievskii, "On the Uniform Convergence of Bieberbach Polynomials in Domains with Piecewise Quasiconformal Boundary," In: Mappings Theory and Approximation of Function, Naukova Dumka Kiev, 1983, pp. 3-18.

[8] V. V. Andrievskii, "Convergence of Bieberbach Polynomials in Domains with Quasiconformal Boundary," 
Ukrainian Mathematical Journal, Vol. 35, No. 3, 1983, pp. 233-236. doi:10.1007/BF01092167

[9] D. Gaier, "Polynoimal Approximation of Conformal Maps," Constructive Approximation, Vol. 14, 1994, pp. 27-40. doi:10.1007/s003659900061

[10] D. Gaier, "On the Convergence of the Bieberbach Polynomials in Regions with Corners," Constructive Approximation, Vol. 4, 1998, pp. 289-305. doi:10.1007/BF02075463

[11] F. G. Abdullayev, "On the Convergence of Bieberbach Polynomials in Domains with Interior Zero Angles," Dokladi Akademii Nauk Ukraine SSR Seria A, Vol. 12, 1989, pp. 3-5.

[12] F. G. Abdullayev, "Uniform Convergence of the Bieberbach Polynomials Inside and on the Closure of Domain in the Complex Plane," East Journal on Approximations, Vol. 7, No. 1, 2001, pp. 77-101.

[13] F. G. Abdullayev and A. Baki, "On the Convergence of Bieberbach Polynomials in Domains with interior zero angles," Complex Variables: Theory and Applications, Vol. 34, No. 2, 2001, pp. 131-143.

[14] D. Israfilov, "Approximation by $p$-Faber Polynomials in the Weighted Simirnov Class $E_{p}(G, w)$ and the Bieberbach Polynomials," Constructive Approximation, Vol. 17, No. 3, 2001, pp. 335-351. doi:10.1007/s003650010030

[15] D. Israfilov, "Uniform Convergence of the Bieberbach Polynomials in Closed Smooth Domains of Bounded Boundary Rotation," Journal of Approximation Theory, Vol. 125, No. 1, 2003, pp. 116-130. doi:10.1016/j.jat.2003.09.008

[16] O. Lehto and K. I. Virtanen, "Quasiconformal Mapping in the Plane," Springer Verlag, Berlin, 1973.

[17] S. Rickman, "Characterization of Quasiconformal Arcs," Annales Academiae Scientiarum Fennicae Mathematica,
1996.

[18] F. G. Abdullayev, "On the Orthogonal Polynomials in Domains with Quasiconformal Boundary," Dissertation, Donetsk (in Russian), 1986.

[19] L. V. Ahlfors, "Lectures on Quasiconformal Mappings," Van Nostrand, Princeton, 1996.

[20] V. V. Andrievskii, V. I. Belyi and V. K. Dzjadyk, "Conformal Invariants in Constructive Theory of Functions of Complex Variable," World Federation Pub., Atlanta, Georgia, 1995.

[21] F. G. Abdullayev, "Uniform Convergence of Generalized Bieberbach Polynomials in Regions with non-zero angles," Acta Mathematica Hungarica, Vol. 77, No. 3, 1997, pp. 223-246.

[22] F. G. Abdullayev, "Uniform Convergence of Generalized Bieberbach Polynomials in Regions with Zero Angles," Czechoslovak Mathematical Journal, Vol. 51, No. 3, 2001, pp. 643-660. doi:10.1023/A:1013796308878

[23] V. I. Belyi and I. E. Pritsker, "On the Curved Wedge Condition and the Continuity Moduli of Conformal Mapping," Ukrainian Mathematical Journal, Vol. 45, No. 6, 1993, pp. 837-844.

[24] V. I. Simirnov and N. A. Lebedev, "Functions of Complex Variable Constructive Theory," MIT Press, Cambridge, 1968.

[25] J. L. Walsh, "Interpolation and Approximation by Rational Functions in the Complex Domain," American Mathematical Society, Rohde Island, 1960.

[26] F. G. Abdullayev and M. Kucukaslan, "On the Convergence of the Fourier Series of Orthonormal Polynmials in the Domain with Piecewise Smooth Boundary," Proceeding of IMM of NAS of Azerbaijan, Vol. 14, No. 22, 2001, pp. 3-13. 